\title{
COMPUTATIONAL STUDIES OF A CHROMATO- GRAPHIC MOVING-BED REACTOR FOR CONSECUTIVE AND REVERSIBLE REACTIONS
}

\author{
Kazuo TAKEUCHI, Terukatsu MIYAUCHI \\ AND THE LATE YUZO URAGUCHI \\ Department of Chemical Engineering, \\ University of Tokyo, Tokyo 113
}

\begin{abstract}
The chromatographic moving-bed reactor is a new device in which catalytic reaction and separation by adsorption take place simultaneously. In this paper, new ways of utilization of the device are investigated to make clear that the following two objects are practicable: (1) the selectivity of the intermediate products in first-order consecutive reactions can be improved compared with that in fixed-bed reactors, and (2) the conversion of products in reversible reactions can exceed the conversion at chemical equilibrium. The para-xylene production process seems to correspond to the second situation.
\end{abstract}

\section{Introduction}

As discussed in the authors' previous papers ${ }^{4,5,7)}$, the chromatographic moving-bed reactor was conceived originally as a device for continuous operation of a chromatographic fixed-bed reactor used for catalyst studies. On the other hand, it can be also considered as an adsorptive alternative of a distillation column accompanied by chemical reactions. Since distillation accompanied by chemical reactions is used for consecutive homogeneous reactions such as, ethylene oxide $\rightarrow$ ethylene glycol $\rightarrow$ polyethylene glycol, and for such homogeneous reversible reactions as esterification, the chromatographic moving-bed reactor may have possibilities in heterogeneous consecutive and reversible reactions.

In the previous papers ${ }^{5,6}$, the basic design procedure and the effect of the exhausting section of the chromatographic moving-bed reactor were discussed for the case of first-order irreversible reactions based on a simple model, according to the so-called quasihomogeneous flow approach. In this approach, it is assumed that each component flows in a homogeneous phase at a superficial velocity $u_{i}=v_{f}-\gamma v_{s}\left(1+K_{i}\right)$, where $v_{f}$ and $v_{s}$ are linear velocities of fluid and solid respectively, and $K_{i}$ is the adsorption equilibrium constant of component $i$. The sign of $u_{i}$ can be either positive, zero or negative, depending on the separation conditions ${ }^{4}$. It was experimentally shown

Received September 10, 1977. Correspondence concerning this article should be addressed to K. Takeuchi. elsewhere ${ }^{8}$, using a model reaction, that catalytic reaction and adsorptive separation do take place simultaneously in this chromatographic moving-bed reactor for the case of catalytic oxidation of carbon monoxide.

Quasi-homogeneous flow approach is based on the assumption that mass transfer rates such as particleto-gas mass transfer rate, intraparticle diffusion and intrinsic adsorption rate are fast compared with reaction rate.

Such an approach is sufficient for primary considerations of basic design and operating conditions of such a reactor/separator. And it is also suited to prediction of maximum performance of this device by such criteria as conversion and surface concentration of product withdrawn from the reactor, even when the effects of mass transfer rates cannot be ignored. In this paper, this approach is employed for two cases and it is shown that selectivity of intermediate products in consecutive reactions can be improved by this device and conversion of the product in reversible reactions can exceed the conversion prescribed by chemical equilibrium.

1. The Chromatographic Moving-Bed Reactor Applied to Consecutive Reactions

\section{1 Theory}

For a series of first-order reactions $\mathrm{F} \stackrel{k_{1}}{\longrightarrow} \mathrm{B} \stackrel{k_{2}}{\longrightarrow} \mathrm{C}$, the basic equations are given by

$$
u_{F}\left(d C_{F} / d x\right)+\gamma k_{r 1} K_{F} C_{F}=0
$$




$$
u_{B}\left(d C_{B} / d x\right)-\gamma\left(k_{r 1} K_{F} C_{F}-k_{r 2} K_{B} C_{B}\right)=0
$$

The equation for component $C$ is not necessary for discussion of selectivity and conversion.

Since discontinuities of the concentration profiles exist at the end of the quasi-homogeneous flows for both the reactant and the product ${ }^{5,6)}$, proper boundary conditions must be adopted at the end of the reactor according to the sign of $u_{i}$.

They are given by

$$
\begin{array}{ll}
C_{F}=\mathrm{C}_{0} & \text { at } x=0 \text { for } u_{F}>0 \\
C_{B}=0 & \text { at } x=0 \text { for } u_{B}>0 \\
& \text { at } x=Z \text { for } u_{B}<0
\end{array}
$$

where $u_{i}=v_{f}-\gamma v_{s}\left(1+K_{i}\right)$

The solutions of Eqs. (1) to (4) are easily given by

$$
\begin{aligned}
& C_{F}=C_{0} \exp \left(\lambda_{1} x\right) \\
& C_{B}=G_{1}\left\{\exp \left(\lambda_{1} x\right)-\exp \left(\lambda_{2} x\right)\right\} \quad \text { for } u_{B}>0
\end{aligned}
$$

and

$$
C_{B}=G_{1} \exp \left(\lambda_{1} x\right)+G_{2} \exp \left(\lambda_{2} x\right) \quad \text { for } u_{B}<0
$$

where

$$
\begin{aligned}
& \lambda_{1}=-k_{1} / u_{F} \\
& \lambda_{2}=-k_{2} / u_{B} \\
& G_{1}=k_{1} C_{0}\left(\lambda_{1}-\lambda_{2}\right) / u_{B} \\
& G_{2}=G_{1} \exp \left\{\left(\lambda_{1}-\lambda_{2}\right) Z\right\}
\end{aligned}
$$

Let $Y_{F B}, Y_{M B}$ and $Y_{M B}^{\prime}$ denote the conversion for the fixed-bed reactor, that for the moving-bed reactor without exhausting section and that for the moving-bed reactor with exhausting section, respectively. They are expressed as follows:

$$
\begin{aligned}
& Y_{F B}=1-\exp \left(-\gamma k_{r 1} K_{F} Z / v_{f}\right) \\
& Y_{M B}=\frac{v_{f}-\gamma v_{s}\left(1+K_{F}\right)}{v_{f}}\left[1-\exp \left\{\frac{-\gamma k_{r 1} K_{F} Z}{v_{f}-\gamma v_{s}\left(1+K_{F}\right)}\right\}\right] \\
& Y_{M B}^{\prime}=1-\exp \left\{\frac{-\gamma k_{r 1} K_{F} Z}{v_{f}-\gamma v_{s}\left(1+K_{F}\right)}\right\}
\end{aligned}
$$

The selectivity of the intermediate product $B$ in the chromatographic moving-bed reactor $\left(S_{M B}\right)$ and that in the conventional fixed-bed reactor $\left(S_{F^{\prime} B}\right)$ can be defined by

$$
\begin{aligned}
S_{M B} & =\text { (Amount of B produced per unit time) } \\
& =\left(C_{B 1}-C_{B 0}\right) u_{B} /\left\{u_{F}\left(C_{0}-C_{F 1}\right)\right\} \\
S_{F^{\prime} B} & =\left(C_{B 1}-C_{B 0}\right) /\left(C_{0}-C_{F 1}\right)
\end{aligned}
$$

where the subscripts 1 and 0 denote the top and the bottom of the rectifying section.

When the quasi-homogeneous flow approach holds, selectivity is not affected by addition of an exhausting section because the required bed length of this section can be considered infinitesimally small compared with that of the rectifying section ${ }^{6}$.

\subsection{Numerical examples and discussion}

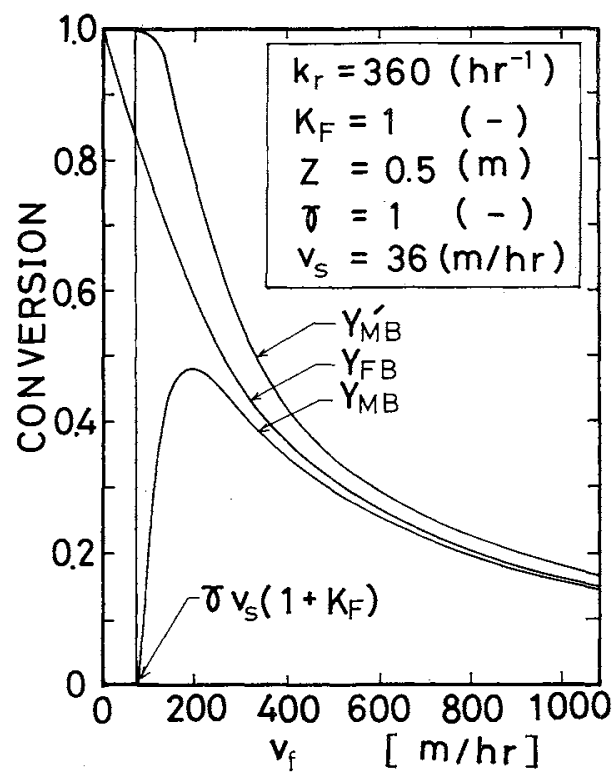

Fig. 1 Numerical example of conversion as functions of $v_{f}$ when other parameters are fixed

Numerical examples of the three kinds of conversion $Y_{F^{\prime} B}, Y_{M B}$ and $Y_{M B}^{\prime}$ are shown in Fig. 1. As discussed in the previous paper ${ }^{6}, Y_{M B}^{\prime}$ is the greatest and $Y_{M B}$ is the smallest among the three kinds of conversion for a given set of parameters. It should be noted that $Y_{M B}$ becomes zero while $Y_{M B}^{\prime}$ is equal to unity at the point where $v_{f}=\gamma v_{s}\left(1+K_{F}\right)$. Figures 2 and 3 illustrate the relations between selectivity and conversion in fixed-bed and moving-bed reactors for given parameters with $v_{f}$ as a sole variable.

The plot of $S_{M B}$ vs. $Y_{M B}$ in Fig. 2 for the chromatographic moving-bed reactor without exhausting section needs some discussion, First, the plot of $S_{M B}$ vs. $Y_{M B}$ shows a loop comprising two parts where the sign of $u_{B}$ is either positive or negative. This is due to the fact that $v_{f}$ has two values for a given $Y_{M B}$, as shown in Fig. 1. Consequently, the conversion in the chromatographic moving-bed reactor without exhausting section cannot exceed a certain maximum value. The point where the loop contacts the abscissa indicates that the selectivity becomes zero when $u_{B}=0$ since in such a case the adsorption wave of component B is stationary in the bed until it is reacted completely.

The plot of $S_{M B}$ vs. $Y_{M B}^{\prime}$ in Fig. 3 is also noteworthy. It also has a point with zero selectivity which corresponds to the condition of $u_{B}=0$. While $S_{F_{B}}$ always approaches zero as $Y_{M B}$ approaches unity, the selectivity approaches a non-zero value as $Y_{M B}^{\prime}$ approaches unity. There are cases where the solid lines in Figs. 2 and 3 are above the broken lines. This shows that the chromatographic moving-bed reactor can be superior to the conventional fixed-bed reactor under equivalent conditions in view of the selectivity of the consecutive reactions. 


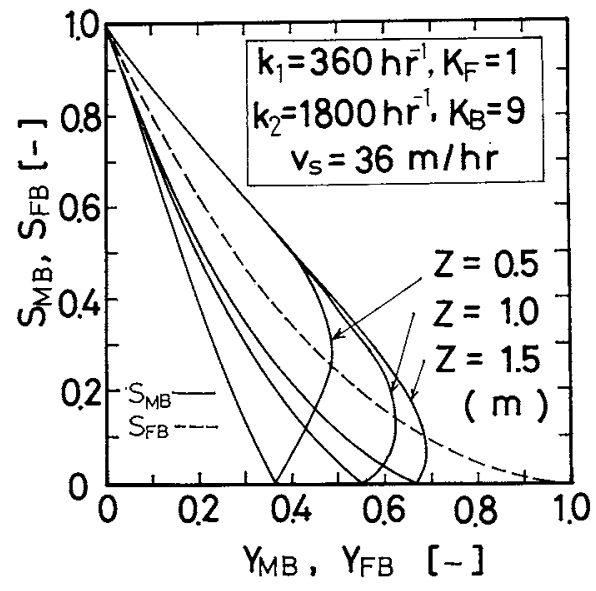

Fig. 2 Numerical example of selectivity vs. conversion for chromatographic moving-bed reactor without exhausting section

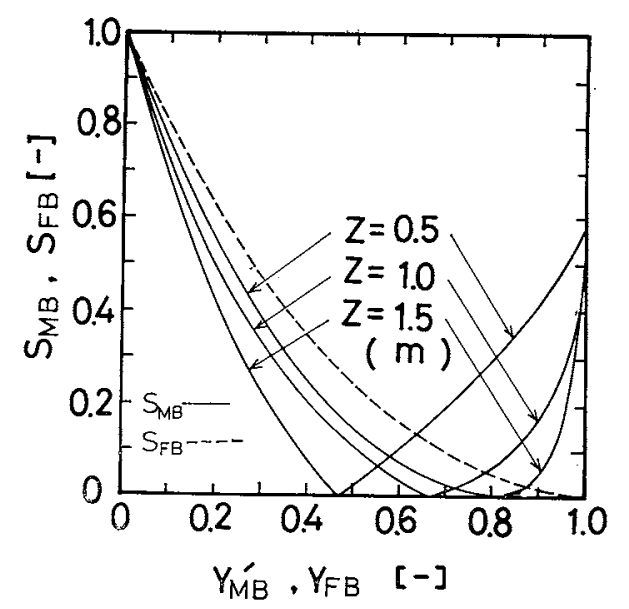

Fig. 3 Numerical example of selectivity vs. conversion for chromatographic moving-bed reactor with exhausting section with same parameters as in Fig. 2

2. Application of the Chromatographic Moving-Bed Reactor to Reversible Reactions-Xylene Isomerization as an Example

\subsection{Theory}

Adopting the quasi-homogeneous flow approach for the case of reversible reactions in the same way as for irreversible ones, the performance of this reactor is examined for the above reactions. The following two additional assumptions are made: (1) both forward and backward reactions are first-order, and (2) the isomers other than para-xylene can be treated as one component. The second assumption is valid since it is reported ${ }^{2)}$ that the selectivities of adsorption between para- and meta-xylene and between para- and orthoxylene are fairly close to each other, and the amount of ortho-xylene and ethylbenzene is relatively small in the commercial multicolumn adsorption process ${ }^{3)}$ for which theoretical treatment is nothing but that of a moving-bed adsorber.
The basic equations for the reversible reaction are written as

$$
\begin{aligned}
& u_{F}\left(d C_{F} / d x\right)+\gamma\left(k_{r 2} K_{F} C_{F}-k_{r 2} K_{P} C_{P}\right)=0 \\
& u_{P}\left(d C_{P} / d x\right)-\gamma\left(k_{r 1} K_{F} C_{F}-k_{r 2} K_{P} C_{P}\right)=0
\end{aligned}
$$

where the subscripts $F$ and $P$ stand respectively for the reactant, which is actually a mixture of meta-xylene, ortho-xylene and ethylbenzene, and for the product para-xylene.

From the separation conditions ${ }^{4)}$, the signs of $u_{F}$ and $u_{P}$ must be different.

The boundary conditions are given by

$$
\begin{aligned}
& C_{F}=C_{0} \text { at } x=0 \\
& C_{P}=0 \text { at } x=Z
\end{aligned}
$$

The solutions of the basic equations are written as

where

$$
\begin{aligned}
& C_{F}=A_{1}+A_{2} \exp (\lambda x) \\
& C_{P}=B_{1}+B_{2} \exp (\lambda x)
\end{aligned}
$$

$$
\begin{aligned}
& \lambda=-\left(k_{1} / u_{F}\right)-\left(k_{2} / u_{P}\right) \\
& A_{1}=C_{0}\left\{1+\left(k_{1} u_{P} / k_{2} u_{F}\right) \exp (-\lambda Z)\right\}^{-1} \\
& B_{1}=C_{0}\left\{\left(k_{2} / k_{1}\right)+\left(u_{P} / u_{F}\right) \exp (-\lambda Z)\right\}^{-1} \\
& A_{2}=C_{0}\left\{1+\left(k_{2} u_{F} / k_{1} u_{P}\right) \exp (\lambda Z)\right\}^{-1} \\
& B_{2}=-C_{0}\left\{\left(u_{P} / u_{F}\right)+\left(k_{2} / k_{1}\right) \exp (\lambda Z)\right\}^{-1}
\end{aligned}
$$

Discontinuities of the concentration profiles exist at the downstream end of the quasi-homogeneous flow for each component as in the case of irreversible reactions. But as long as the discussion is based on fluxes, no further consideration of discontinuities is necessary. Nor need the effect of backward reaction from para-xylene to meta-xylene in the exhausting section be considered, because the required bed length of the exhausting section is sufficiently small when the quasi-homogeneous flow approach holds. Thus the exhausting section only plays a role for the recovery of unreacted materials.

\section{2 Numerical calculations and discussion}

To illustrate the design method for a chromatographic moving-bed reactor with exhausting section according to the model in the previous section, the values for $K_{F}, K_{P}, k_{1}$, and $k_{2}$ are necessary. Mixture of a known isomerization catalyst and an adsorbent used for xylene separation for use in liquid phase may be a realistic candidate for such a catalyst/adsorbent combination since their operating temperatures are close to each other, while the possibility exists of developing more favorable catalyst adsorbent combinations.

According to the liquid chromatographic data of de Rosset et al. ${ }^{3}$, the retention time of para-xylene relative to non-adsorbing nonane is found to be 1.8 at their operating temperature, which is considered somewhat below $200^{\circ} \mathrm{C} . \quad K_{P}$ is obtained as follows.

Relative retention time $=1+\gamma\left(1+K_{P}\right) /(1+\gamma)$

When $\gamma$ is estimated at about 1.0, $K_{P}=1.6$. A patent 
for zeolitic adsorbent for para-xylene adsorption ${ }^{2)}$ shows that the adsorption selectivity between paraand meta-xylene lies between 3.6 and 4.7. When the value of 4.7 is chosen for $\alpha_{p-m}$, which is equal to $K_{P} / K_{F}, K_{F}$ can be obtained as 0.34 .

On the other hand, the forward reaction rate constant $k_{1}$ from meta- to para-xylene by a zeolitic catalyst for liquid-phase isomerization is reported to be about $1 \mathrm{hr}^{-1}$ at $170^{\circ} \mathrm{C}^{1)}$. The reaction rate constant for isomerization of ortho-xylene to para-xylene is reported to be considerably less than the above value. The superficial chemical equilibrium constant $K_{\text {chem }}$ based on assumption (2) in the previous section 2.1 is given by

$$
K_{\text {chem }}=k_{1} / k_{\mathrm{2}}=\left(X_{P}\right)_{\mathrm{eq}} /\left\{1-\left(X_{P}\right)_{\mathrm{eq}}\right\}
$$

where $\left(X_{P}\right)_{e q}=0.21$ at $170^{\circ} \mathrm{C}$, and $K_{c \text { nem }}$ is obtained as 0.26 . Consquently, the backward reaction rate constant $k_{2}$ is calculated to be $4.85 \mathrm{hr}^{-1}$.

The mixture of catalyst and adsorbent mentioned above can be treated as a single catalyst/adsorbent. When the packing conditions and other geometrical factors are not changed by mixing, the parameters for this catalyst/adsorbent can be obtained by such simple modifications as $\left(K_{i}\right)_{\mathrm{mix}}=K_{i} \phi$ and $\left(k_{j}\right)_{\mathrm{mix}}=k_{j}(1-\phi)$ where $i=F$ or $P, j=1$ or 2 and $\phi$ is the volume fraction of the adsorbent in the solid mixture. We arbitrarily choose $\phi=0.5$.

A para-xylene production rate of 100,000 tons per year or $1.2 \times 10^{4} \mathrm{~kg} / \mathrm{hr}$ is considered for calculation as a standard scale of production. Let $Y_{M B}^{\prime}$ and $Q_{P 0}$ denote the conversion in the chromatographic movingbed reactor with an exhausting section, and the flux of the product withdrawn at the bottom of the exhausting section. They are written as

$$
\begin{aligned}
& Y_{M B}^{\prime}=\left(C_{0}-C_{F 1}\right) / C_{0} \\
& Q_{P 0}=-C_{P 0} u_{P}
\end{aligned}
$$

The condition that the separation can take place is written as

$$
\gamma v_{s}\left\{1+\left(K_{F}\right)_{\mathrm{mix}}\right\}<v_{f}<\gamma v_{s}\left\{1+\left(K_{P}\right)_{\mathrm{mix}}\right\}
$$

As in the case of irreversible reaction discussed previously $\left.^{5}\right), v_{s}$ is fixed to be $25 \mathrm{~m} / \mathrm{hr}$ in this calculation. Then $Y_{M B}^{\prime}$ and $Q_{P 0}$ can be calculated as functions of $v_{f}$ and $Z$ as shown in Figs. 4 and 5 . Figure 5 shows that $Q_{P 0}$ has a maximum for given $Z$ and is zero at both ends of the separation conditions given by inequality (32). When $v_{f}$ is chosen close to the lower limit $\gamma v_{s}\left\{1+\left(K_{F}\right)_{\mathrm{mix}}\right\}$, the conversion is high enough to exceed the equilibrium conversion $\left(X_{P}\right)_{\mathrm{eq}}$ in fixed beds. But in this case $Q_{P_{0}}$ is small, as shown in Fig. 5, and the required bed diameter to satisfy the given production rate becomes larger. Liquid linear velocity $v_{f}$ should not be chosen between the maxima and $\gamma v_{s}\{1+$ $\left.\left(K_{P}\right)_{\text {mix }}\right\}$ in Fig. 5, because for every fixed value of $Q_{P_{0}} / C_{0}$ there is another possible choice of $v_{f}$ between

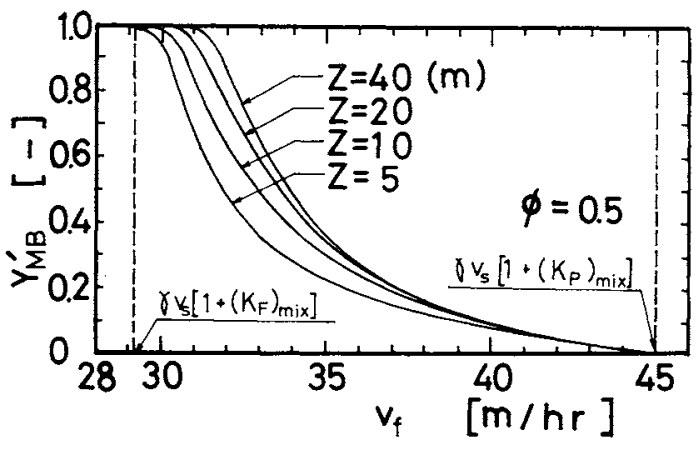

Fig. 4 Conversion $Y_{M B}^{\prime}$ vs. $v_{f}$ for chromatographic moving-bed reactor with exhausting section

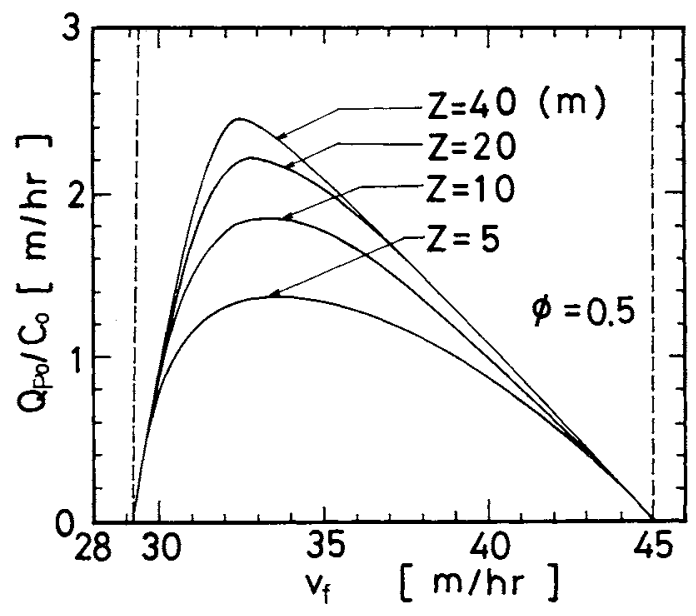

Fig. 5 Withdrawal rate of product $Q_{P 0}$ vs. $\boldsymbol{v}_{f}$

the maxima and $\gamma v_{s}\left\{1+\left(K_{F}\right)_{\mathrm{mix}}\right\}$, which is superior in view of the conversion and pressure drop consideration. We may choose $v_{f}=32.5 \mathrm{~m} / \mathrm{hr}$ for all the values of $Z$, which gives roughly the maxima of $Q_{P 0} / C_{0}$ in Fig. 5. When $v_{f}$ and $Q_{P 0}$ are given, the radius of the reactor can be calculated from the relation

$$
Q_{P_{0}} \alpha \pi R^{2}=F_{x}=1.2 \times 10^{4} \mathrm{~kg} / \mathrm{hr}
$$

where $F_{x}$ is the required production rate of paraxylene. $C_{0}$ is arbitrarily chosen to be $650 \mathrm{~kg} / \mathrm{m}^{3}$.

In Table 1, comparison is made between the conversion for the chromatographic moving-bed reactor with an exhausting section and that for the fixed-bed reactor of same geometry and $v_{f}$. The latter is calculated by the relation

$$
\ln \left[\left\{1-\left(X_{P}\right)_{\mathrm{eq}}\right\} /\left\{X_{F}-\left(X_{F}\right)_{\mathrm{eq}}\right\}\right]=\left(k_{1}+k_{2}\right) Z / v_{f}
$$

If the bed length of the chromatographic moving-bed reactor is chosen to be $5 \mathrm{~m}$, the conversion will be almost fourfold compared with the corresponding fixed-bed reactor of the same geometry and $v_{f}$. While this calculation is based on assumptions such as quasi-homogeneous flow approach as the most favorable case for this reactor/separator, it is important to note that the fractional conversion of the product can exceed the value that is normally restricted by chemical equilibrium relations for fixed-bed reactors. 
Table 1 Comparison of performance of a chromatographic moving-bed reactor with that of a conventional fixed-bed reactor

\begin{tabular}{|c|c|c|c|c|c|c|}
\hline \multirow[b]{2}{*}{$\begin{array}{l}Q_{P 0} / C_{0} \\
{[\mathrm{~m} / \mathrm{hr}]}\end{array}$} & \multirow[b]{2}{*}{$\begin{array}{c}v_{f} \\
{[\mathrm{~m} / \mathrm{hr}]}\end{array}$} & \multirow[b]{2}{*}{$\begin{array}{c}Z \\
{[\mathrm{~m}]}\end{array}$} & \multirow[b]{2}{*}{$\begin{array}{c}R \\
{[\mathrm{~m}]}\end{array}$} & \multirow[b]{2}{*}{$\begin{array}{c}\text { Bed volume } \\
\pi R^{2} Z \\
{\left[\mathrm{~m}^{3}\right]} \\
\end{array}$} & \multicolumn{2}{|c|}{ Conversion } \\
\hline & & & & & $\begin{array}{c}\text { Moving bed } \\
Y_{M B}^{\prime} \\
{[-]}\end{array}$ & $\begin{array}{c}\text { Fixed bed of the same } \\
\text { geometry and } v_{f}\end{array}$ \\
\hline 1.34 & 32.5 & 5 & 3.31 & 172 & 0.42 & 0.12 \\
\hline 1.82 & 32.5 & 10 & 2.84 & 253 & 0.55 & 0.18 \\
\hline 2.20 & 32.5 & 20 & 2.58 & 418 & 0.67 & 0.20 \\
\hline 2.46 & 32.5 & 40 & 2.44 & 748 & 0.76 & 0.21 \\
\hline
\end{tabular}

\section{Conclusion}

It is thus shown numerically, for the first time, that the selectivity of the intermediate product in consecutive reactions and the conversion of the product in reversible reactions such as xylene isomerization can be improved by use of the chromatographic movingbed reactor.

\section{Nomenclature}

$A_{1} \quad=$ defined by Eq. (24)

$A_{2} \quad=$ defined by Eq. (26)

$B_{1} \quad=$ defined by Eq. (25)

$B_{2} \quad=$ defined by Eq. (27)

$C=$ concentration in bulk

$C_{0} \quad=$ feed concentration

$G_{1} \quad=$ defined by Eq. (10)

$G_{2} \quad=$ defined by Eq. (11)

$K_{i} \quad=$ adsorption equilibrium constant based on unit pore volume

$K_{\mathrm{chem}}=k_{1} / k_{2}$, chemical equilibrium constant

$k_{1} \quad=\gamma k_{r 1} K_{F}$

$k_{2}=\gamma k_{r 2} K_{B}$ or $\gamma k_{r 2} K_{P}$

$k_{r 1}=$ surface reaction rate constant for $F \rightarrow B$ or $F \rightarrow P$

$k_{r 2} \quad=$ surface reaction rate constant for $B \rightarrow C$ or $P \rightarrow F$

$\begin{array}{ll}Q_{P 0} & =\text { flux of product withdrawal } \\ S_{F B} & =\text { selectivity in fixed bed as defined by }\end{array}$ Eq. (16)

$S_{M A B} \quad=$ selectivity in moving bed as defined by Eq. (15)

$u_{i} \quad=v_{f}-\gamma v_{s}\left(1+K_{i}\right)$, linear velocity of quasi-homogeneous flow of component $i \quad[\mathrm{~m} / \mathrm{hr}]$

- linear fluid velocity

$=$ linear solid velocity

$=C_{F} / C_{0}$ $\left[\mathrm{kg} / \mathrm{m}^{2} \cdot \mathrm{hr}\right]$
$X_{P} \quad=\quad=\mathrm{C}_{P} / C_{0}$

$x \quad=$ distance from feed location

$Y_{F B} \quad=$ fixed-bed conversion

$Y_{M B} \quad=$ moving-bed conversion without exhausting section

$Y_{M B}^{\prime} \quad=$ moving-bed conversion with exhausting section

$=$ bed length

$[-]$

$Z \quad=$ exhausting

$\alpha \quad=$ void fraction of bed

$\alpha_{p-m}=$ adsorption selectivity between para- and meta-xylene

$=$ porosity of particle

$=(1-\alpha) \beta / \alpha$

$=$ defined by Eq. (23)

$=$ defined by Eq. (8)

$=$ defined by Eq. (9)

$=$ volume fraction of adsorbent in solid mixture

$$
\begin{array}{r}
{[\mathrm{m}]} \\
{[-]} \\
{[-]} \\
{[-]} \\
{[\mathrm{m}]} \\
{[-]} \\
{[-]} \\
{[-]} \\
{[-]} \\
{\left[\mathrm{m}^{-1}\right]} \\
{\left[\mathrm{m}^{-1}\right]} \\
{\left[\mathrm{m}^{-1}\right]} \\
{[-]}
\end{array}
$$

$\langle$ Subscripts〉

$\begin{array}{rll}{[-]} & \text { eq } & =\text { at chemical equilibrium } \\ {[-]} & F & =\text { reactant } \\ {\left[\mathrm{hr}^{-1}\right]} & P & =\text { product of reversible reaction } \\ {\left[\mathrm{hr}^{-1}\right]} & 1 & =\text { at } x=Z \\ & 0 & =\text { at } x=0\end{array}$

[hr- $\left.{ }^{-1}\right] \quad$ Literature Cited

[hr-1] 1) Chutoransky Jr., P. and F. G. Dwyer: "Molecular Sieves", p. 540, Am. Chem. Soc. (1973).

2) Otani, S.: Japan patent 48-66583.

[-] 3) de Rosset, A. J. et al.: Ind. Eng. Chem., Process Des. Dev., 15, 261 (1976).

[-] 4) Takeuchi, K. and Y. Uraguchi: J. Chem. Eng. Japan, 9, 164 (1976).

5) Takeuchi, K. and Y. Uraguchi: ibid., 9, 246 (1976).

6) Takeuchi, K. and Y. Uraguchi: ibid., 10, 72 (1977).

7) Takeuchi, K. and Y. Uraguchi: ibid., 10, 297 (1977).

8) Takeuchi, K. and the late Y. Uraguchi: ibid., 10, 455 (1977). 